
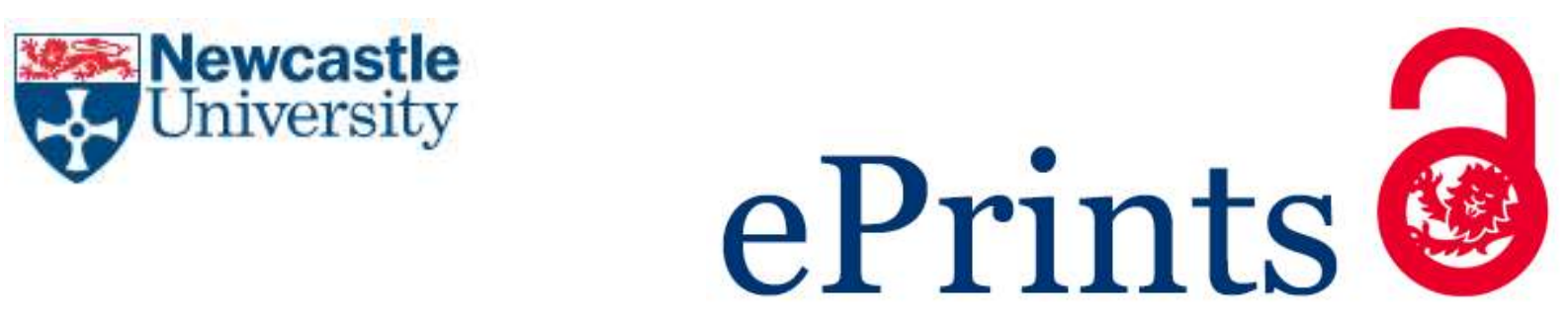

Hamilton J, Ingham B, McKinnon I, Parr J, Tam L, Le Couter A. Mental capacity to consent to research? Experiences of consenting adults with intellectual disabilities and/or autism to research. British Journal of Learning Disabilities 2017

\title{
Copyright:
}

This is the peer reviewed version of the following article: Hamilton J, Ingham B, McKinnon I, Parr J, Tam L, Le Couter A. Mental capacity to consent to research? Experiences of consenting adults with intellectual disabilities and/or autism to research. British Journal of Learning Disabilities 2017, which has been published in final form at https://doi.org/10.1111/bld.12198 . This article may be used for noncommercial purposes in accordance with Wiley Terms and Conditions for Self-Archiving.

Date deposited:

$02 / 08 / 2017$

Embargo release date:

17 July 2018

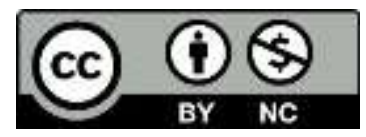

This work is licensed under a Creative Commons Attribution-NonCommercial 3.0 Unported License 
This is the accepted version of the following article:

Hamilton J, Ingham B, McKinnon I, Parr JR, Tam LY-C, Le Couteur A. Mental capacity to consent to research? Experiences of consenting adults with intellectual disabilities and/or autism to research. Br J Learn Disabil. 2017;00:1-8. https://doi.org/10.1111/bld.12198, which has been published in final form at http://onlinelibrary.wiley.com/journal/10.1111/(ISSN)1468-3156/earlyview.

This article may be used for non-commercial purposes in accordance with the Wiley Self-Archiving Policy [ http://olabout.wiley.com/WileyCDA/Section/id-828039.html ].

\section{Mental capacity to consent to research? Experiences of consenting adults with intellectual disabilities and autism to research}

Jahnese Hamilton NIHR Clinical Research Network (Mental Health), Northumberland Tyne and Wear NHS Foundation Trust, UK, Barry Ingham Psychological Services, Northgate Hospital, Northumberland Tyne and Wear NHS Foundation Trust, UK, lain McKinnon Institute of Health and Society, Newcastle University, UK and Forensic Services, Northumberland Tyne and Wear NHS Foundation Trust, UK, Jeremy R Parr Institute of Neuroscience, Newcastle University, UK, Louise Yuen-Chong Tam NIHR Clinical Research Network (Mental Health), Northumberland Tyne and Wear NHS Foundation Trust, UK, Ann Le Couteur Institute of Health and Society, Newcastle University, UK.

Correspondence: Jahnese Hamilton, NIHR CRN NENC, Wolfson Research Centre, C.A.V., NE4 5PL. Tel.01912081367. Fax.01912081385. E-mail Jahnese.Hamilton@ntw.nhs.uk

\section{Accessible Summary}

- The NHS Constitution states that all patients should have opportunity to take part in approved research

- This study asked clinical researchers how they include people with intellectual disabilities and/or autism in research

- Many barriers were identified that relate to making mental capacity judgements

- Many researchers agreed new resources that support consent and capacity judgements for research would be helpful and gave some ideas about what could help

\footnotetext{
Abstract

Background: Adults with intellectual disabilities and autism are often excluded from participating in health and healthcare research. Understanding study information, which is an important aspect of demonstrating capacity to give informed consent, can be a particular challenge. This study surveyed clinical researchers to discover (i) their experiences of assessing mental capacity for research; (ii) what methods they used to facilitate the inclusion of adults with intellectual disabilities and/or autism; and (iii) their views about a proposal to develop new resources to facilitate mental capacity judgements with adults with intellectual disabilities and/or autism for informed consent for research.
} 
Methods: Clinical researchers in North East England who conduct research with NHS patients with intellectual disabilities and/or autism were invited to participate in a 22 item self-completed semi-structured questionnaire survey, either online or on paper.

Results: Twenty-one clinicians completed the survey (response rate 30.4\%). Participants reported on 18 research studies which included people with intellectual disabilities and/or autism. In many studies people who lacked capacity to give informed consent were excluded, and often shortcuts were taken in judging capacity. Limited adaptations to support capacity were used. Respondents welcomed the proposal of developing assistive resources that could support capacity judgements and consent to research.

Conclusions: To improve access to research for people with intellectual disabilities and/or autism, researchers need robust methods to facilitate informed consent and mental capacity judgements. Future research should determine which assistive resources show potential to support informed consent and capacity decisions, and whether such resources could improve inclusion in research.

\section{Introduction}

The National Health Service (NHS) Constitution for England (NHS, 2015) states that the NHS belongs to the people and includes the key principles of equality of access, excellence in standards of care through service provision and staff support, and commitment to research. However, adults with intellectual disabilities and/or autism are affected by a range of health inequalities including problems with standards of care, service provision, and appropriate implementation of the Mental Capacity Act (2005) (Emerson \& Baines, 2010; Heslop et al., 2014). Research offers the potential to improve evidence based healthcare, but there is a substantial lack of primary research and systematic reviews on many aspects of the health of adults with intellectual disabilities and/or autism (Anderson et al., 2013; Mukaetova-Ladinska, Perry, Baron, \& Povey, 2012; Robertson, Hatton, Baines, \& Emerson, 2015). There is also emerging evidence that people with intellectual disabilities and autism are frequently excluded from healthcare research. For example, one review reported that only 6/300 (2\%) of clinical trials included adults with intellectual disabilities (Feldman, Bosett, Collet, \& Burnham-Riosa, 2014). Further Brooker et al. (2015) identified that 11/56 (20\%)of Randomised Controlled Trials and 7/97 (7\%) of cohort studies actively excluded adults with intellectual disabilities by inclusion/exclusion criteria and $41 / 56$ (73\%) of randomised controlled trials and 52/97 (54\%) of cohort studies passively excluded through the lack of accessible consent or recruitment procedures.

When adults with intellectual disabilities were asked about the challenges they perceive in taking part in research, they reported that understanding study information and filling in consent forms were specific difficulties (Lennox et al., 2005). Adults with autism also identified that a number of different factors including consideration of the individual's cognitive, behavioural and sensory needs are important for enhancing accessibility. They recommended opportunities to discuss the research; using visual aids and prompts that help with communication and remembering; having research meetings in relaxed and quiet venues; and having carers or companions available to assist communication and reassurance (Haas et al., 2016).

In seeking to include adults across the autism spectrum, current large-scale cohort research undertaken by the authors JP, ALC, BI \& JH (The Adult Autism Spectrum Cohort-UK study (ASC-UK), http://research.ncl.ac.uk/adultautismspectrum/, UKCRN ID 18481), has identified a number of challenges to the recruitment of individuals with greater support needs. These have included how best to make judgements about capacity and the identification of appropriate consultees for adults who may lack capacity to give informed consent. In summary, there are multiple challenges faced when seeking to include adults 
with intellectual disabilities and/or autism in trials and cohort studies which can inform health and healthcare needs.

One specific challenge in research is the responsibility of obtaining valid informed consent and alongside this, judging mental capacity to give consent. Valid informed consent for research ensures: information has been effectively communicated and understood; decisions about participation are recognised as voluntary; benefits are appropriately weighed up against risks; and the potential participant's capacity to give their own informed consent is recognised (Health Research Authority, 2017; National Institute for Health Research, 2016; World Health Organization, 2005). These core responsibilities of the appropriately qualified researcher who obtains informed consent from people who may lack capacity, are also outlined in detail in the Mental Capacity Act's (2005) supporting documentation, entitled 'Conducting research with people not having the capacity to consent to their participation: a practical guide for researchers' (Dobson, 2008):

The researcher's role, in addition to reaching a judgement about the ability of a participant to give consent, is also to consider the balance of the benefit of participation with an evaluation of 'proportionate risk'; (Dobson, 2008, p. 8) and

In terms of decision-making under the Mental Capacity Act, the key question for the researcher is, does the person have the capacity to consent (or refuse) at the time the decision needs to be made? (Dobson, 2008, p. 11)

Accordingly researchers carry an ethical and legal responsibility, when obtaining informed consent, to judge the capacity of participants and the quality of the consent itself. However, for studies involving adults with intellectual disabilities, there are difficulties in putting these guidelines into practice: Jepson (2015) reports that judgements about capacity can fluctuate when recruiting potential participants with intellectual disabilities who for example exhibit good social communication strategies but have underlying difficulties with memory, or alternatively need further time to communicate their thoughts.

UK legislation covering inclusion of adults who may lack capacity is country specific: For England and Wales the "Mental Capacity Act" (2005); for Scotland the "Adults with Incapacity (Scotland) Act" (2000); and for Northern Ireland the "Mental Capacity Act (Northern Ireland)" (2016); with Clinical Trials of investigational medicinal products governed separately in UK-wide legislation ("The Medicines for Human Use (Clinical Trials) Regulations," 2004). The existence of separate regulatory frameworks may bring challenges to researchers, examples of which have been considered by Shepherd (2016). However common principles exist across the jurisdictions, notably the requirements for ethical review, for benefits to outweigh potential risks, for individuals' needs to be put before the needs of science or society, and for legal representatives or consultees to be consulted on behalf of persons who lack capacity to give specific informed consent. In the event of potential participants being judged to lack capacity, decisions about participation or nonparticipation are dependent on the recommendation from the appropriate consultee (such as a relative) or guardian (Scotland) or legal representative (for Clinical Trials) combined with the willingness of the potential participant (Department of Health, 2008; "Mental Capacity Act," 2005). Capacity judgements must be considered carefully: In England and Wales, and recently Northern Ireland, the Mental Capacity Acts (2005, 2016) direct that all reasonable steps are taken to support capacity, "A person is not to be treated as unable to make a decision unless all practicable steps to help him to do so have been taken without success" ("Mental Capacity Act," 2005, p. 1). This process of making capacity judgements therefore requires researchers to do preliminary work around supporting capacity.

There is a small literature that considers strategies to support capacity to make decisions for research, such as individualised communication (Cameron \& Murphy, 2007), adapting consent materials (Taua, Neville, \& Hepworth, 2014), and using participatory approaches such as a recursive approach to consent (Cook \& Inglis, 2012). However these strategies do not in themselves determine capacity to give informed consent. Two 5-6 
item questionnaires have been developed to assist the process of determining capacity for research consent with people with intellectual disabilities (Arscott, Dagnan, \& Kroese, 1998; Horner-Johnson \& Bailey, 2013). However there is little evidence in the literature that these questionnaires are widely used and they also make limited use of supportive strategies to empower informed decision making. Furthermore the effectiveness of strategies intended to support or judge capacity has not been adequately evaluated. There is a clear need to understand how clinical researchers in the UK currently approach informed consent and mental capacity judgements with adults with intellectual disabilities and/or autism, and to identify what else is required to support these judgements.

\section{Research Questions}

To understand current clinical research practice for establishing capacity within informed consent processes with adults with intellectual disabilities and/or autism, we undertook a survey of clinical researchers in one of the UK's largest mental health and disability NHS Trusts. Our aim was to explore what specific challenges the respondents identified and what strategies they used to manage these challenges. The survey addressed three research questions: (i) what are clinical researchers' experiences, if any, of assessing mental capacity for research?; (ii) what methods do clinical researchers currently employ to facilitate the inclusion of adults with intellectual disabilities and/or autism in research; and (iii) would clinical researchers like further resources to support informed consent and mental capacity judgements and what suggestions do they have for facilitating this process?

\section{Methods}

\section{Participants}

Clinical researchers working with adults with intellectual disabilities and/or autism in NHS hospital and community outpatient settings in North East England were identified using opportunistic sampling methods through local networks and asked to pass on the invitation to participate to other relevant colleagues. 69 researchers were documented as approached and 21 (30.4\% response rate) completed the survey.

\section{Materials}

The survey comprised 22 semi-structured questions with open-ended text boxes. The survey questions were derived from review of the literature (specifically the existing guidance, policy and legislation on mental capacity) and framed around the research objectives. The questionnaire contained 5 sections: An introduction to the survey; Characteristics of participants and research reported on (six items); decisions relating to mental capacity (six items); informed consent procedures and adaptations including involvement of others (five items); views on the development of resources to support capacity judgements and informed consent processes (five items) (see Supplement 1).

Survey forms could be completed anonymously: participants' demographics, employment roles, and study names were not collected to protect anonymity. The survey was initially piloted amongst the study team, leading to minor wording modifications and six additional clarifying questions.

\section{Procedure}

A favourable ethical opinion from Newcastle University (reference: 7772/2016) and NHS Service Evaluation approval (reference: SER-16-024) was obtained. Potential participants were approached in person/by email. 
Consent was voluntary and implied through questionnaire return. Participants were asked to complete the survey on their own, on paper (SAE envelope provided for return), or online (Qualtrics survey platform: https://www.qualtrics.com/).

\section{Analysis}

A descriptive analysis was undertaken where the free text responses were examined by a process of familiarization which involved reading and re-reading the data (JH \& LYCT). Then, in an iterative cycle the authors $(\mathrm{JH}, \mathrm{BI}, \mathrm{IM}, \mathrm{LYCT}, \mathrm{ALC})$ discussed the topics and organisation, and agreed on the selection of representative quotes. The data was organised according to topics relating to each research question, and descriptive statistics were calculated. Descriptive statistics were organised with illustrative quotes (see results). Interpretation of these findings together with consideration of the strengths and limitations of the study are presented in the discussion.

\section{Results}

Questionnaires took approximately 15 to 20 minutes to complete. 18/21 (86\%) of survey participants each reported experience of up to five research studies involving adults with intellectual disabilities and/or autism. 3/21 (14\%) participants reported no direct research with adults with intellectual disabilities or autism in the last 10 years, but had clinical or research experiences which enabled them to respond to the second half of the questionnaire (regarding the proposal to develop assessment tools). Of the 18 studies discussed, only one was a Clinical Trial of an Investigational Medicinal Product (CTIMP) (a study that was unable to recruit any participants). Two studies were clinical 'case-studies'. Two studies involved taking saliva samples and conducting cognitive tasks. Three studies were evaluations of group based interventions/assessments. Three studies were described as qualitative research, and seven studies involved primarily face-to-face or postal questionnaires.

\section{Question 1: What are clinical researchers' experiences, if any, of assessing mental capacity for research?}

Two topics were identified:

\section{Inclusion/exclusion criteria for including participants who lack capacity in the study}

Five of the 18 studies (28\%) included participants who lacked capacity through agreement with their consultee. Participation of those who lacked capacity was dependent upon participant engagement with the researchers and the relevant tasks; if the participant seemed unhappy then the researcher would not include them in the study.

[If they lacked capacity] they were included and consent could be sought from carer/parent if client was able to do some tasks (QP1).

One participant was willing to sit whilst we described the study but was unable to engage in tasks at the most basic level \& would probably have become distressed \& aggressive if we'd continued (QP2).

13 studies (72\%) reported that potential participants who lacked capacity were excluded: justifications were given such as appropriateness of involvement or the quality of the data. In these circumstances, however, it was not apparent that formal capacity assessments had been made prior to exclusion of the individuals concerned: 
The study could not recruit participants who lacked capacity to consent for themselves as we needed them to be able to answer questionnaires for themselves (QP15).

If it was considered that clients lacked capacity to take part they would not be included in the study (QP6).

Other reasons for exclusion rested on opinions from Research Ethics Committees, despite researchers recognising meaningfulness in participation for such individuals:

We did not include them [...] the ethics committee deemed that as we were able to do the research with men who could consent [...] (Although as stated previously, we did indeed end up including one man who understood that he did not have to take part but would struggle with what the research was about and the concept of informed consent throughout the research) (QP12).

There was a participant whose data we did not include due to concerns about capacity and understanding during the completion of the questions. We continued with the process as it was felt like the most appropriate course of action to take, to preserve the participant's dignity, however this information was not included (QP14).

\section{Person(s) involved in making capacity judgements for informed consent}

10 studies (56\%) reported the researchers having a role in judging the capacity of a participant to consent, which could have been a shared role. For eight (44\%) of studies researchers described this responsibility being given to others such as clinicians or family members.

Researcher completed a brief assessment of capacity prior to commencing the questionnaires (QP14)

Clinicians only referred participants they believed had capacity to consent. Researchers with the help of family members or carers [also] determined this (QP2).

Clinicians only approached young people who they deemed to not have a significant intellectual disability and were able to answer and consent for themselves (QP15).

In summary, the studies included people with intellectual disabilities and/or autism, however participants who were thought, by the researchers and or by others, to lack capacity were often excluded, possibly without any formal assessment taking place. While many researchers recognised their role in judging capacity to give informed consent, this decision was deferred to clinicians or family members in just under half of the studies reported on.

Question 2: What methods do clinical researchers currently employ to facilitate the inclusion of adults with intellectual disabilities and/or autism in research?

The data showed two topics aligned to this question:

\section{Adaptations to support inclusion}

All 18 studies (100\%) reported that they made adaptations to materials to support understanding. This was mainly using easy read versions and/ or verbalising the information. Family, carers and inpatient staff were often involved in some way with these procedures. In a small number of cases other communication aides such as Talking Mats (Cameron \& Murphy, 2007) (one study) or use of a CD (one study) were mentioned. The 
benefits of easy read documents were occasionally questioned, and one survey respondent recognised the challenge of understanding research for people who had had no experience of research.

Easy read info with pictures. Most participants relied more on verbal description \& the paperwork was more of a distraction than being particularly useful (QP2).

[We used] Verbal cues, easy read, talking mats, communication aids (QP9).

Consent forms were also provided and participants were encouraged to share these with people at home and ask any questions they might have (QP14).

Verbal cues, easy read participant information forms with a $C D$ where all the info was read out and the person could keep listening to it alongside the easy read. Plain English consent forms. These were useful for some, but not all. The main issue was that when we did the recruitment the men did not really understand the notion of research and so could not imagine what they were, in their words 'letting themselves in for' (QP12).

\section{Method of obtaining consent}

16 of the studies (89\%) reported that the protocol required written consent. Only two studies (11\%) allowed the use of 'witnessed verbal consent'. Some researchers identified participant engagement with tasks as non-verbal communication of willingness to participate, although this was for participants who were judged to lack capacity to give informed consent.

Implied consent occurred throughout as tasks were either undertaken or not. Notes were written in study paperwork and patient records (QP2).

In summary all research studies described making some adaptations to support understanding, but these adaptations were often limited. For obtaining evidenced informed consent very few studies provided alternatives to written consent.

\section{Question 3: Would clinical researchers like further resources to support informed consent and mental} capacity judgements and what suggestions do they have for facilitating this process?

The data showed two topics aligned to this question:

\section{Would the researcher like to see further resources developed?}

Although $15 / 21$ participants (71\%) in the survey agreed that developing tools to assist making capacity assessments would be helpful, four participants (19\%) saw no requirement for further resources. Two (10\%) of the participants were unsure. Supporters believed further resource development could help support research in the field of intellectual disabilities and/or autism.

- Yes extremely useful! [...] Especially anything over and above MCA [(Mental Capacity Act)]. -Helpful for both research development and participation as need to consider steps to assess capacity in research protocol (QP6).

Potentially lack of clear methodology for assessing capacity is a deterrent to undertaking research in this field (QP1). 
Having a best-practice evidence-based tool to assess would help researcher go about this process in the best way e.g. what to ask and how to ask it. It would also ensure that the process is more reliable and valid (QP6).

Yes, especially with regard to transparency in legal obligations and getting proposals passed in first place (QP17).

Those who did not want further tools to aid capacity judgements or resources cited feeling comfortable with existing guidelines and practices. There was also concern that certain types of tools could lead to a distance between the researcher and the participants.

I don't think this decision is significantly different from any other decision that we may have to assess capacity for, so I don't see that it needs a separate tool (QP10)

It would depend. I think it's not all about assessment, but about engagement and relationship building in order to develop understandings. It's the way we, as prospective researchers, find ways to enable people to learn about what it means to be in 'this' research project in ways that are meaningful for them. I don't think a tool can do this as it suggests there is something that can be imposed, or given to, but it is all about building knowing together and that will be different according to the type of research, the type of engagement and the current understandings of the person we would like to be involved (QP12)

Similarly, those who were unsure cited concerns about the versatility of such resources in meeting different demands.

I'm thinking that the issue of consent is bound up with issues that are not easily reducible to an assessment tool [...] I'm thinking that assessing reading level and comprehension (which are to my mind some of the simpler issues in the task that you are seeking to undertake - e.g. the issue of the power gradient, voice, agency and autonomy are much more complex issues to address) require at least the of MDT for each person. [...] I wonder if an ethical framework with principles of action and response might work. I think it is highly worthy, timely and important to think these things through (QP13).

\section{Suggestions for what to include in resources}

Regardless of opinions on developing assistive resources, respondents gave a range of suggestions for what they thought would be useful in the process of assessing mental capacity for research. Fifteen respondents (71\%) gave specific suggestions: Seven (33\%) suggested supported decision making / decision aids; four (19\%) suggested using visual aids; four (19\%) suggested re-confirming consent at the end of data collection; three (14\%) suggested including family/supporter/informants; and three (14\%) specifically suggested 'person-centred options'.

Supported decision making, visual and decisional aids. Re-consent at the end of data collection (PP4).

Decision aids such as talking mats (QP5).

Also having a family member/known face (if available) to facilitate the communication (QP7).

In summary there were mixed responses to developing assessment tools, although the majority of researchers stated they would find this helpful. The suggestions emphasised the potential need for a variety of person-centred options that can respond to individual needs. 


\section{Discussion}

This study found that people with intellectual disabilities and/or autism who are perceived to lack capacity to understand information or communicate decisions are frequently excluded from research with potential relevance to them, either actively or passively. The researchers who completed our survey also frequently reported delegating decisions about capacity to clinicians or family members (which is not in keeping with UK guidelines for best practice, or existing legislation (Dobson, 2008)). The methods and tools used that might support capacity were frequently limited to strategies such as verbalising information and using easy read materials. More research is needed to develop assistive resources and to derive evidence of efficacy for a wider range of personalised and person-centred strategies that show potential to help people with intellectual disabilities and/or autism understand research, and to make informed decisions about their participation. Many of the clinical researchers who completed this survey welcomed a proposal for the development of resources to support consent and capacity judgements and all respondents provided suggestions for what to include. Examples included decision aids, visual aids, revisiting consent after data collection, including family and carers, and embracing a range of person centred approaches.

\section{Making judgements about mental capacity}

Following Jepson (2015) it was anticipated that researchers might raise concerns about the risk of fluctuating capacity during the course of a study. This issue is rarely mentioned in the survey responses, one reason for this may be because the respondents report that many of the studies had excluded participants who lacked capacity. This practice of exclusion could lead to only approaching participants who are deemed to be verbally fluent and have a mild intellectual disability/or are relatively high functioning. Given that capacity is decision specific, there is the risk that participants who have limitations in verbal fluency and moderate to profound needs are systematically denied opportunities to demonstrate capacity. Furthermore researchers and clinicians may be reluctant to approach potential participants who could subsequently be assessed as lacking capacity. Recent literature challenges this "institutional exclusion of seldom-heard groups" (Turnpenny et al., 2015 pg.8.), citing the potential risk of a severe lack of representation of people with moderate to profound intellectual disabilities in research literature (lacono, 2006).

One argument raised by the survey respondents for excluding adults who lack capacity, is the premise that it is unethical to include adults lacking capacity if the research question can genuinely be answered through recruitment of capacitous individuals alone. In contrast however, two respondents reported ethical dilemmas considering the exclusion of participants from activities they had begun or in which their peers were participating, when it became apparent that they lacked a full understanding of the research methods. The interplay between ethical research practice and the existing governing legislation is further complicated when considering how commitment to international equality laws affect clinical research. The UN Convention on the Rights of Persons with Disabilities (2006) ratified by the UK in 2009, establishes a commitment to fundamental freedoms of persons with disabilities (including those lacking capacity); to enabling autonomy; and to making reasonable adaptations to ensure inclusion in all areas of public life and healthcare. The applicability and influence of this international agreement on research practice across the UK merits further consideration by researchers and policy makers.

\section{Supporting inclusion}

To support informed decision making, a limited number of strategies to aid accessibility of relevant information were used in all reported studies. This predominantly referred to easy read information sheets/consent forms and verbalising information. Infrequently, other visual prompts such as 'Talking Mats' (Cameron \& Murphy, 2007) were mentioned. Carers and relatives are also recognised as potentially 
supportive to communication. Often however participation depended upon a potential research participant having the ability to sign a written consent form. This shows an over-reliance on ability to read and write as a prerequisite to participation in many types of health research. Consent to take part in research that is not a medicinal trial can be accepted in writing, orally, or non-verbally (Health Research Authority, 2017). Additionally, medicinal trials are permitted to adapt consent processes to include giving consent orally in the presence of at least one witness and recorded in writing for participants who are unable to sign or to mark a document separately ("The Medicines for Human Use (Clinical Trials) Regulations," 2004).

It is understandable that researchers may prefer to formalise consent using written consent procedures. However such restrictions are likely to mean that people with literacy challenges are being 'dis-abled' from participating in research when no legally recognised alternatives are considered. Given the frequency of the use of written consent to evidence informed consent in this survey, this topic would benefit from further investigation.

\section{Developing mental capacity assessment tools for research}

Overall there was strong support from the participants for the proposal of specific mental capacity assessment tools to support decision making for research amongst those with intellectual disabilities. Some respondents questioned the practicalities of whether such tools would help or hinder capacity assessments in research (e.g. if the tools restricted best practice). A consensus emerged that the process requires more than simply the use of a checklist of questions to set out the constructs of capacity (as provided by Arscott et al., 1998; Horner-Johnson \& Bailey, 2013). Healthcare providers, researchers and funders are internationally urged to develop understanding of how assistive devices can be utilised to support understanding, communication and inclusion of people with intellectual disabilities and/or autism (Tomlinson et al., 2014). The respondents in this study suggest that decision aids, visual aids, re-consenting at the end of data collection, including carers, and using person-centred approaches are valuable starting points for the development of such resources for informed consent and capacity decisions in research.

\section{Limitations}

The findings of this study are limited by the size and geographical scope of the sample drawn from the North East of England. Furthermore as study names were not collected to protect anonymity, it is possible that respondents reported experiences on the same studies conducted locally. However a variety of different types of studies were reported on by the participants. Legislation covering mental capacity for Scotland or for Northern Ireland differs to England and Wales; given these differences in legislation and the timing of these Acts, it is possible that researchers in other jurisdictions will report different experiences. Differences between legislation for Clinical Trials of Investigational Medicinal Products and other forms of research may also be impacting on practices involving the inclusion (or exclusion) of those who lack capacity in research: details of this has not been detailed in this paper but is discussed by Shepherd (2016). While this study has not gone further into contrasting the differences between legislations the current sample of clinical researchers surveyed offers a rich account of the experiences of researchers working under the Mental Capacity Act (2005) in England. This detailed feedback adds to the evidence that this is a complex topic requiring careful consideration and investigation.

\section{Future research}

The findings from this survey provide support and guide our research proposal to develop resources which support specific capacity judgements for informed consent for research by adults with intellectual disability and/or autism. Detailed recommendations recognise that resources must meet the need to support mental capacity and decision making for research, prior to making a potential lacking capacity judgement ("Mental Capacity Act," 2005 principle 2). Future research should be conducted in partnership with adults with 
intellectual disability and/or autism to identify and evaluate the utility of potential assistive resources to (a) support understanding and decision making by adults with intellectual disability and/or autism, and (b) indicate when a 'lacking capacity' judgement is appropriate. Following the identification of relevant resources, the feasibility, acceptability, reliability, validity and effectiveness of these 'tools' will need to be carefully investigated to evaluate how they can be recommended for use in health and healthcare research. It is possible that greater confidence in addressing issues of capacity may lead to an increase in reported rates of inclusion of adults with intellectual disabilities and/or autism in research.

\section{Acknowledgements}

We are grateful to Northumberland Tyne and Wear NHS Foundation Trust for Research Capability Funding 2016/17 - RCF1617-010 to enable these research questions to be investigated.

We are also grateful to the UK autism research charity Autistica for funding the Adult Autism Spectrum Cohort-UK (ASC-UK), which has guided us towards the current research questions on capacity and consent. See http://research.ncl.ac.uk/adultautismspectrum/ for further details.

\section{References}

Adults with Incapacity (Scotland) Act (2000).

Anderson, L. L., Humphries, K., McDermott, S., Marks, B., Sisarak, J., \& Larson, S. (2013). The state of the science of health and wellness for adults with intellectual and developmental disabilities. Intellectual and Developmental Disabilities, 51, 385-398. doi: 10.1352/1934-9556-51.5.385

Arscott, K., Dagnan, D., \& Kroese, B. S. (1998). Consent to psychological research by people with an intellectual disability. Journal of Applied Research in Intellectual Disabilities, 11, 77-83.

Brooker, K., van Dooren, K., Tseng, C. H., McPherson, L., Lennox, N., \& Ware, R. (2015). Out of sight, out of mind? The inclusion and identification of people with intellectual disability in public health research. Perspectives in Public Health, 135, 204-211. doi: 10.1177/1757913914552583

Cameron, L., \& Murphy, J. (2007). Obtaining consent to participate in research: the issues involved in including people with a range of learning and communication disabilities. British Journal of Learning Disabilities, 35, 113-120. doi: 10.1111/j.1468-3156.2006.00404.x

Cook, T., \& Inglis, P. (2012). Participatory research with men with learning disability: informed consent. Tizard Learning Disability Review, 17, 92-101. doi: 10.1108/13595471211218875

Department of Health. (2008). Guidance on nominating a consultee for research involving adults who lack capacity to consent. London: Department of Health, Scientific Development and Bioethics Division Retrieved from http://www.hra.nhs.uk/documents/2013/07/guidance-on-nominating-a-consulteefor-research-involving-adults-who-lack-capacity-to-consent.pdf.

Dobson, C. (2008). Conducting research with people not having the capacity to consent to their participation: a practical guide for researchers. Leicester: British Psychological Society.

Emerson, E., \& Baines, S. (2010). Health inequalities \& people with learning disabilities in the UK. Online: Improving Health and Lives: Learning Disabilities Observatory.

Feldman, M. A., Bosett, J., Collet, C., \& Burnham-Riosa, P. (2014). Where are persons with intellectual disabilities in medical research? A survey of published clinical trials. Journal of Intellectual Disability Research, 58, 800-809. doi: 10.1111/jir.12091 
Haas, K., Costley, D., Falkmer, M., Richdale, A., Sofronoff, K., \& Falkmer, T. (2016). Factors influencing the research participation of adults with autism spectrum disorders. J Autism Dev Disord, 46, 1793-1805. doi: 10.1007/s10803-016-2708-6

Health Research Authority. (2017). Principles of consent: general principles and role of participant information sheets. Retrieved 07/02/2017, from http://www.hradecisiontools.org.uk/consent/principles-general.html

Heslop, P., Blair, P. S., Fleming, P., Hoghton, M., Marriott, A., \& Russ, L. (2014). The confidential inquiry into premature deaths of people with intellectual disabilities in the UK: a population-based study. Lancet, 383, 889-895. doi: 10.1016/S0140-6736(13)62026-7

Horner-Johnson, W., \& Bailey, D. (2013). Assessing understanding and obtaining consent from adults with intellectual disabilities for a health promotion study. Journal of Policy and Practice in Intellectual Disabilities, 10, 1-10. doi: 10.1111/jppi.12048

Iacono, T. (2006). Ethical challenges and complexities of including people with intellectual disability as participants in research. Journal of Intellectual and Developmental Disability, 31, 173-179. doi: $10.1080 / 13668250600876392$

Jepson, M. (2015). Applying the Mental Capacity Act to research with people with learning disabilities. British Journal of Learning Disabilities, 43, 128-134. doi: 10.1111/bld.12122

Lennox, N., Taylor, M., Rey-Conde, T., Bain, C., Purdie, D. M., \& Boyle, F. (2005). Beating the barriers: recruitment of people with intellectual disability to participate in research. Journal of Intellectual Disability Research, 49, 296-305.

The Medicines for Human Use (Clinical Trials) Regulations (2004).

Mental Capacity Act (2005).

Mental Capacity Act (Northern Ireland) (2016).

Mukaetova-Ladinska, E. B., Perry, E., Baron, M., \& Povey, C. (2012). Ageing in people with autistic spectrum disorder. International journal of geriatric psychiatry, 27, 109-118. doi: 10.1002/gps.2711

National Institute for Health Research. (2016). Good Clinical Practice (GCP) reference guide. Leeds: NIHR Clinical Research Network Coordinating Centre Retrieved from http://www.nihr.ac.uk/ourfaculty/documents/GCP\%20Reference\%20Guide.pdf.

NHS. (2015). The NHS Constitution for England. England: Department of Health Retrieved from https://www.gov.uk/government/publications/the-nhs-constitution-for-england.

Robertson, J., Hatton, C., Baines, S., \& Emerson, E. (2015). Systematic reviews of the health or health care of people with intellectual disabilities: a systematic review to identify gaps in the evidence base. Journal of Applied Research in Intellectual Disabilities 2015, 28, 455-523. doi: 10.1111/jar.12149

Shepherd, V. (2016). Research involving adults lacking capacity to consent: the impact of research regulation on 'evidence biased' medicine. BioMed Central Medical Ethics, 17, 1-8. doi: 10.1186/s12910-0160138-9

Taua, C., Neville, C., \& Hepworth, J. (2014). Research participation by people with intellectual disability and mental health issues: an examination of the processes of consent. Int J Ment Health Nurs, 23, 513524. doi: 10.1111/inm.12079

Tomlinson, M., Yasamy, M. T., Emerson, E., Officer, A., Richler, D., \& Saxena, S. (2014). Setting global research priorities for developmental disabilities, including intellectual disabilities and autism. Journal of Intellectual Disability Research, 58, 1121-1130. doi: 10.1111/jir.12106

Turnpenny, A., Caiels, J., Crowther, T., Richardson, L., Whelton, B., Beadle-Brown, J., . . Rand, S. (2015). Developing an easy read version of the Adult Social Care Outcomes Toolkit (ASCOT). Canterbury: QORU Quality and Outcomes of Person-Centred Care Policy Research Unit.

United Nations Convention on the Rights of Persons with Disabilities (2006).

World Health Organization. (2005). Handbook for good clinical research (GCP): guidance for implementation. Geneva: World Health Organization Retrieved from http://www.who.int/iris/handle/10665/43392. 